\title{
Ámbito de Hogar y Movimientos del Zorrillo (Mephitis macroura) en la Época Lluviosa en el Campus de Zamorano, Honduras
}

\author{
José Manuel Mora ${ }^{1}$, Gerardo Montes de Oca ${ }^{2}$ y Baleshka R. Brenes ${ }^{3}$
}

Resumen. El zorrillo (Mephitis macroura) existe en el sudoeste de Estados Unidos, México y Centroamérica pero la información disponible de la especie en esta última región es limitada. Se identificaron aspectos generales de la ecología del zorrillo en el campus de la Escuela Agrícola Panamericana (Universidad Zamorano), Honduras. Aunque se rastreó por telemetría a ocho individuos por periodos variables de tiempo, solo se determinaron los ámbitos de hogar de dos machos adultos, un macho joven y una hembra adulta para un período de cuatro meses. El tamaño del ámbito de hogar de estos individuos varió entre 3.4 y 32.4 ha (promedio $=16.8$ ha). El tamaño del ámbito de hogar de los tres machos fue mayor que el de la hembra y el ámbito de hogar de los dos machos adultos fue mayor que el del macho joven. No existió intersección entre las áreas de los dos machos adultos pero el ámbito de hogar de la hembra se encontró dentro del de los machos. Las ubicaciones de los zorrillos fueron en áreas verdes (jardines con árboles), edificios, cercas vivas, muros de piedras, escombros, madrigueras subterráneas y basureros. Los muros de piedra y los edificios estudiantiles son refugios para el zorrillo ya que proveen espacios adecuados para su descanso, protección, alimentación y crianza. La hembra rastreada tuvo cuatro crías, las cuales fueron observadas al final del estudio (septiembre 2009).

Palabras clave: Carnívoros, mofetas, radiolocalizaciones, radiotelemetría.

\section{Home Range and Movements of the Hooded Skunk (Mephitis macroura) in the Rainy Season on the Zamorano Campus, Honduras}

\begin{abstract}
The hooded skunk (Mephitis macroura) occurs in the southwest United States, Mexico and Central America but information available for the species in the latter region is limited. Several general aspects of the ecology of this skunk were identified in the campus of Zamorano University (Escuela Agrícola Panamericana), Honduras. Although eight individuals were tracked by telemetry for variable periods of time only the home range of two males, a young male and an adult female were determined for a period of four months. Home range size of these individuals varied between 3.4 and 32.4 ha (mean = $16.8 \mathrm{ha}$ ). Home range size of the three males was larger than the female's and the home range size of the two adult males was larger than that of the young male. Intersection did not exist between the areas of both adult males but the home range of the female was inside the male home ranges. Skunk localizations were in green areas (gardens with trees), buildings, shrubs, rock walls, rubble, underground burrows, and trash-bins. Rock walls and student buildings are skunk refuges since they provide spaces adequate for resting, protection, feeding, and reproduction. The tracked female had four babies which were observed at the end of the study (September, 2009).
\end{abstract}

Key words: Carnivores, radiolocalizations, radiotelemetry.

\section{Introducción}

Existen 12 especies de zorrillos o mofetas las (Mephitidae, Carnivora) y cuatro se encuentran en Centroamérica. El zorrillo (Mephitis macroura) es un mamífero fácilmente detectable debido a que produce un líquido muy oloroso cuando se siente amenazado (Mora, 2000). El cuerpo es pequeño y los individuos presentan variaciones en la coloración. Pueden tener la espalda negra con dos líneas blancas en los costados,

\footnotetext{
${ }^{1}$ Centro Zamorano de Biodiversidad, Departamento de Ambiente y Desarrollo, Escuela Agrícola Panamericana, Zamorano, Honduras. Correo electrónico jmora@zamorano.edu

${ }^{2}$ Plazas de San Buenaventura, San Marciano 123. Toluca, Estado de México. CP: 50110, México. Correo electrónico gerardo.mdos@gmail.com

31809 Mayo St Apt.1, Commerce, Texas 75428, Estados Unidos. Correo electrónico Baleshka@ gmail.com
} 
la espalda blanca con dos líneas blancas en los costados o completamente negros con un mechón blanco en la punta de la cola (Reid, 2009). El peso varía entre 0.4 - $2 \mathrm{~kg}$ (Mora, 2000) y las hembras son generalmente $15 \%$ más pequeñas que los machos (Hwang y Larivière, 2001). Esta especie se encuentra distribuida en el sudoeste de Estados Unidos, México y Centroamérica (Reid, 2009).

Este zorrillo es nocturno y generalmente solitario, pero varios individuos se pueden reunir, sin agresión, en un sitio de alimentación (Mora, 2000). Según este último autor el zorrillo es omnívoro a pesar de ser miembro del orden Carnivora (Mammalia). Durante el día duerme en grietas de rocas, hoyos, madrigueras o entre arbustos (Hwang y Larivière, 2001). La reproducción se da normalmente desde mediados de febrero a finales de marzo (Hwang y Larivière, 2001). La gestación es de 65 días y el tamaño de la camada es de tres a cinco crías, aunque algunas veces pueden nacer hasta ocho cachorros (Mora, 2000).

Los zorrillos expelen por el ano n-butyl mercaptan, una sustancia venenosa que ataca al sistema nervioso si se ingiere y podría causar la muerte (De la Rosa y Nocke, 2000). Recibirla directamente puede causar ceguera temporal y vómito y el olor es difícil de remover (De la Rosa y Nocke, 2000).

El ámbito de hogar es el área donde un individuo realiza sus actividades diarias (Burt, 1943). Éste no es toda el área que un animal cubre durante su tiempo de vida, si no el área donde normalmente se mueve. Los movimientos fuera del área normal utilizada, no deben de ser considerados como parte del ámbito de hogar (White y Garrott, 1990). El término ámbito de hogar es utilizado en este informe para describir dos aspectos de la ecología del zorrillo. El primero, es el mapa básico de ubicaciones obtenidas como producto del seguimiento a los animales. El segundo es la estimación numérica del área usada por el animal o tamaño del ámbito de hogar.

Las poblaciones de carnívoros están disminuyendo en el mundo debido al crecimiento de las poblaciones humanas $\mathrm{y}$, principalmente, a las características biológicas intrínsecas de los miembros del orden (Cardillo et al., 2004). No obstante, el zorrillo es bastante avistado en la Escuela Agrícola Panamericana, Zamorano, Honduras, por lo que aparentemente es abundante. Esto se puede deber a ciertas condiciones favorables que la especie encuentra en el campus y a la protección que la vida silvestre goza en Zamorano debido a la filosofía ambientalista que identifica a la institución.

Debido a ello se procedió a estudiar esta especie por telemetría en el campus, particularmente el ámbito de hogar y los requerimientos ecológicos básicos del zorrillo. Por lo tanto, este estudio puede aportar información valiosa para la protección de ésta u otras especies en sitios con mayor amenaza.

\section{Materiales y Métodos}

El área de estudio fue el campus central de la Escuela Agrícola Panamericana, Zamorano, en el Valle del Yeguare, Departamento de Francisco Morazán, Honduras. El campus está a a $30 \mathrm{~km}$ al este de Tegucigalpa, a $800 \mathrm{msnm}$ y está localizada dentro del bosque seco tropical. Esta área incluye canchas deportivas, áreas verdes con jardines y árboles, potreros, zonas residenciales, edificios, cercas vivas, muros de piedra y es atravesada por la carretera panamericana.

Se hicieron observaciones nocturnas con linternas para determinar los sitios que más frecuentaban los zorrillos para determinar donde colocar las trampas para su captura. Se usaron 10 trampas tipo Tomahawk que fueron colocadas en varios lugares del campus. El cebo usado incluyó carne de res, pollo y cerdo.

Para colocar los collares se usaron guantes de cuero para evitar mordidas y rasguños. Se utilizó un plástico protector y anteojos plásticos para acercarse a las trampas donde estaban los zorrillos, para evitar ser afectado por la secreción anal expulsada por los zorrillos cuando se sienten amenazados.

Los zorrillos fueron identificados por el sexo y peso, y se les colocó un radio collar. Cada radio tenía una frecuencia diferente (Cuadro 1). Cada zorrillo fue liberado en el mismo sitio donde fue capturado. La telemetría es un sistema de monitoreo a distancia que se basa en ondas radiales transmitidas desde un emisor y captadas por un recibidor (Rabinowitz, 2003). Los radios transmisores se colocan en los animales a seguir y así se localizan y a la vez se monitorean las actividades de los individuos (Cochran, 1987). 
Cuadro 1. Identificación (nombre dado) de cada zorrillo (Mephitis macroura) capturado y características relevantes para el trabajo de telemetría en el campus de Zamorano, Honduras, 2009.

\begin{tabular}{llrccl}
\hline $\begin{array}{l}\text { Nombre } \\
\text { del zorrillo }\end{array}$ & Sexo & $\begin{array}{c}\text { Peso } \\
(\mathbf{g})\end{array}$ & $\begin{array}{c}\text { Frecuencia }(\text { VHF) } \\
\text { radio }\end{array}$ & Días de rastreo & Observaciones \\
\hline Bale & Hembra & 750 & 149.921 & 10 & Perdido \\
Banquita & Hembra & 690 & 149.961 & 2 & Se quitó el collar \\
Campeón & Macho & 1,100 & 149.881 & 3 & Se quitó el collar \\
Chiriquí & Macho adulto & 1,010 & 149.961 & 102 & Rastreado \\
Felipe & Macho juvenil & 450 & 150.000 & 59 & Rastreado \\
Lanik & Hembra & 745 & 149.842 & 13 & Se quitó el collar \\
Leuca & Hembra preñada & 1,550 & 149.842 & 60 & Rastreado \\
Mex & Macho adulto & 1,200 & 149.881 & 107 & Rastreado \\
Peludo & Macho & 1,340 & & 0 & Solo pesado \\
\hline
\end{tabular}

Los zorrillos se localizaron en la noche para determinar el ámbito de hogar de cada individuo. Fueron localizados con un recibidor (RX-TLNX) y dos antenas, la Yagi de recepción a distancias mayores y la omnidireccional para distancias cortas. La antena detecta la señal de cada radio transmisor que es seguida en el recibidor según la frecuencia seleccionada. Cada vez que se localizó un zorrillo se tomó su ubicación con un GPS. Los datos se registraron en coordenadas UTM (Universal Transverse Mercator) y el datum WGS84. Las ubicaciones se refieren al punto que fue registrado en el lugar donde se encontró al individuo.

El tiempo que se dedicó al trabajo con trampas y la captura fue de 47 noches, con un promedio de cuatro noches/trampa por zorrillo. Posterior a la captura y ya con los radios colocados se trabajó en bloques de dos horas cada noche (seleccionados al azar) entre las 6:00 pm y las 6:00 am. Este trabajo se realizó de esa manera durante un periodo de cuatro meses. Durante una semana por mes, de los cuatro meses de estudio, se intensificaron las localizaciones de los zorrillos y se trabajó seis horas por noche.

Una vez finalizado el trabajo de campo se calculó el ámbito de hogar de cada individuo por medio de polígonos mínimos de frecuencias (PMF) al 95\% de confiabilidad, con el programa Biotas 1.03.3 alpha. Con el mismo programa se calculó también la curva de área acumulada para cada zorrillo. Así mismo se identificó el uso del hábitat de los individuos con la ayuda del programa de Sistemas de Información Geográfica ArcGIS 9.3 y se utilizaron ortofotos del área de estudio para una mejor interpretación de los datos.

\section{Resultados}

Se capturaron nueve zorrillos, cinco machos y cuatro hembras, de los cuales se pudo seguir trabajando con cuatro hasta el final del estudio (Cuadro 1). Tres zorrillos se quitaron el collar, Campeón y Banquita al siguiente día de su captura y Lanik lo tuvo 13 días. Posteriormente los radios fueron encontrados y reutilizados para colocarlos en nuevos individuos. Es por esto que la frecuencia de los radios en el Cuadro 1 se encuentra repetida. Una de las hembras (Bale) con collar no se pudo seguir rastreando ya que la señal se dejó de escuchar 10 días después de haberla liberado.

El área total que abarcó el estudio fue 72.8 ha, donde se registraron 140 ubicaciones de los zorrillos. Los ámbitos de hogar de los cuatro zorrillos rastreados variaron de 3.4 a 32.4 ha. El mayor ámbito de hogar fue el de Chiriquí con un área de 32.4 ha, a diferencia de Leuca que tuvo el menor con 3.4 ha. Entre estos extremos se determinó 11.1 ha para Felipe y 20.4 ha para Mex. Algunos de los polígonos de frecuencia de los zorrillos estudiados presentan intersección entre ellos (Figura 1). El polígono de mayor extensión es el de Chiriquí que tuvo algún grado de interacción con el de Felipe (Figura 1). 


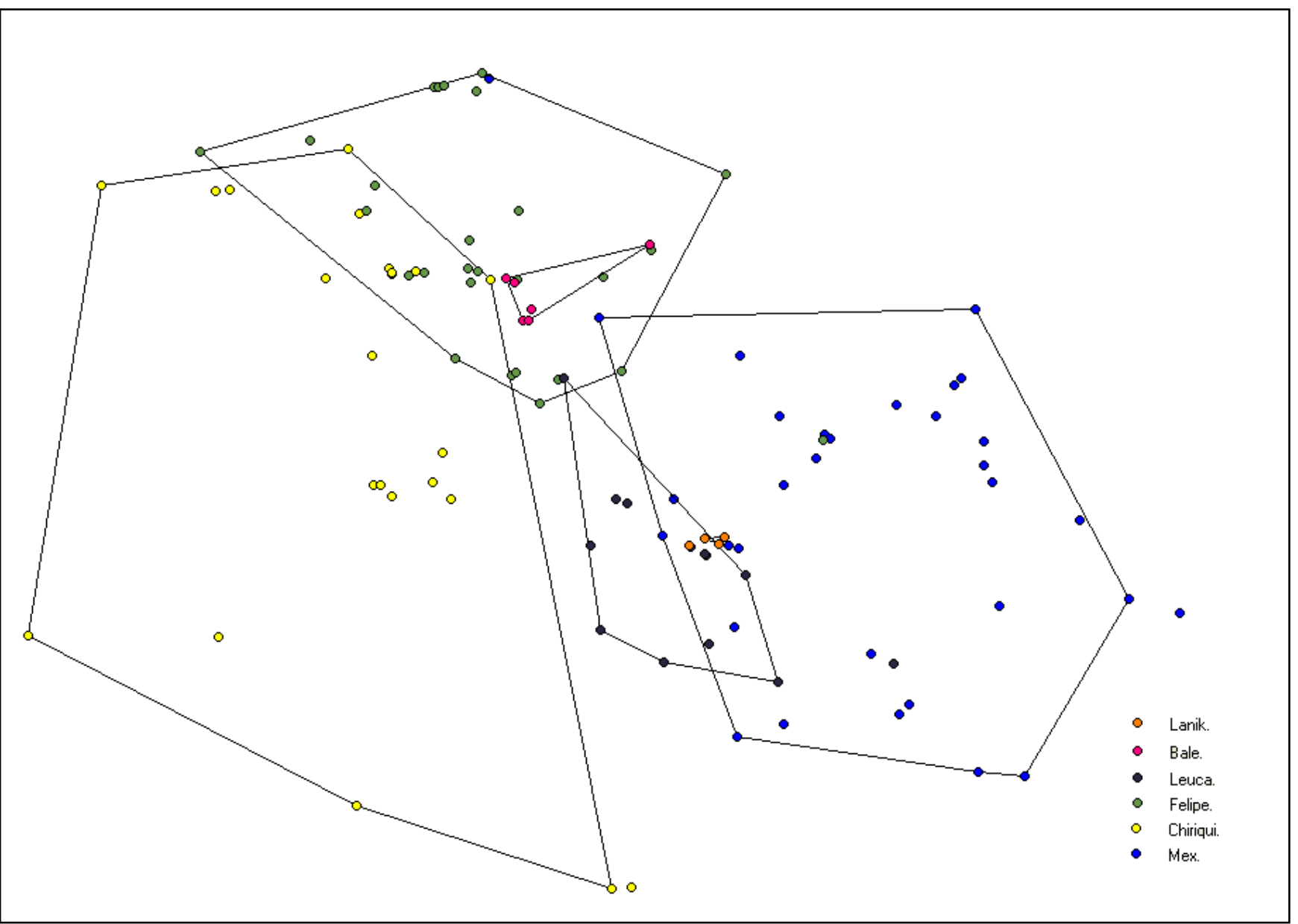

Figura 1. Intersección de los polígonos de frecuencia de los zorrillos (Mephitis macroura) hembras denominados Leuca, Lanik y Bale con el de los machos estudiados (Felipe, Chiriquí y Mex) en el campus de la Escuela Agrícola Panamericana, Zamorano, Honduras, 2009.

La curva de área acumulada de los individuos rastreados presentó, por lo general, un incremento en el tamaño del ámbito de hogar con relación al número de localizaciones registradas. El área del ámbito de hogar de Chiriquí tuvo una relación proporcional con el número de localizaciones registradas (Figura 2), esta tendencia fue similar para Mex y Felipe. La curva del área acumulada de Leuca presentó una tendencia diferente a la del resto de los individuos, ya que no aumentó durante las nueve localizaciones (Figura 3).

Las ubicaciones de Chiriquí se encuentran cerca de árboles y muros de roca en su mayoría, así mismo se dieron localizaciones en áreas abiertas más lejanas del área residencial del campus central. El ámbito de hogar de Chiriquí fue de 32.4 ha (Figura 4).

La distribución de localizaciones de Felipe dentro del campus incluyó edificios y áreas con árboles. Su área de ámbito de hogar se concentró en el campus central (edificio principal Zemurray, figura 5) al principio del estudio pero en las últimas localizaciones se había alejado de su centro de actividad. Con ello se aumentó el tamaño del área del ámbito de hogar a 11.1 ha (Figura 5). 


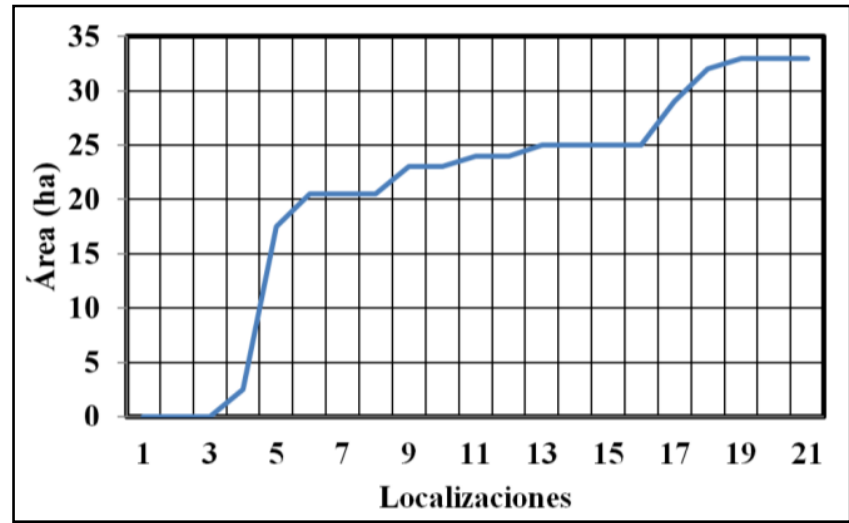

Figura 2. Curva de área acumulada del ámbito de hogar del zorrillo (Mephitis macroura) nombrado Chiriquí estudiado en el campus de la Escuela Agrícola Panamericana, Zamorano, Honduras, 2009.

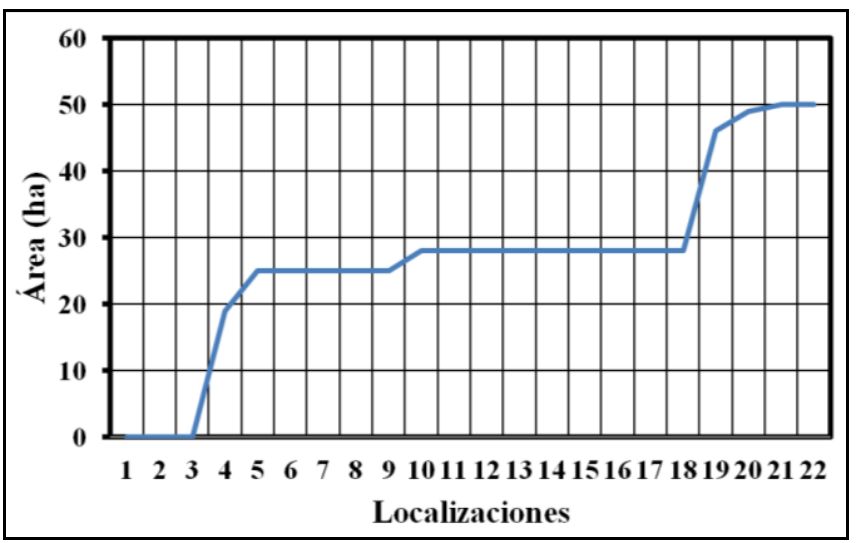

Figura 3. Curva de área acumulada del ámbito de hogar del zorrillo (Mephitis macroura) nombrado Leuca estudiado en el campus de la Escuela Agrícola Panamericana, Zamorano, Honduras, 2009.

Leuca es una hembra que se encontraba preñada al momento de ser capturada. Ella se encontró del 1 al 23 de agosto de 2009 dentro de las madrigueras, todas las veces que fue localizada (Figura 6). Tuvo una camada de cuatro crías, tres tienen el fenotipo de su madre (espalda blanca) y una de ellas es de un fenotipo distinto (espalda negra). El ámbito de hogar de Leuca fue de 3.4 ha. El mayor número de ubicaciones de Leuca fue debajo de un camino de piedras, donde tenía su madriguera de crianza (representada por un círculo en la figura 6).

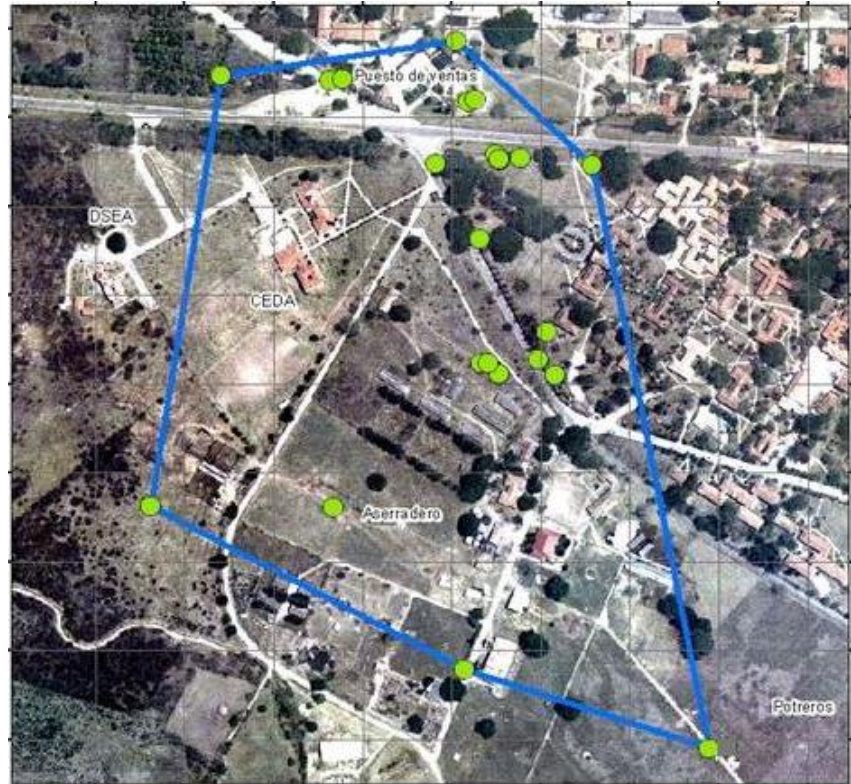

Figura 4. Polígono de frecuencias y localizaciones del zorrillo (Mephitis macroura) nombrado Chiriquí en el campus de la Escuela Agrícola Panamericana, Zamorano, Honduras, 2009.

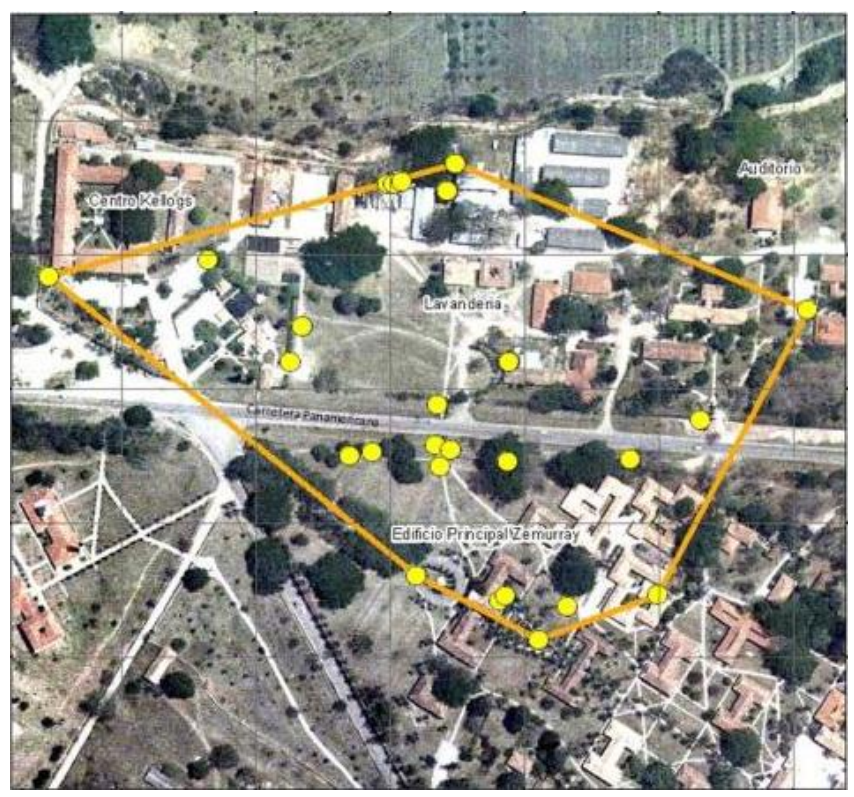

Figura 4. Polígono de frecuencias y localizaciones del zorrillo (Mephitis macroura) nombrado Felipe en el campus de la Escuela Agrícola Panamericana, Zamorano, Honduras, 2009. 
El área del ámbito de hogar de Mex fue de 20.41 ha y se localizó en diferentes sitios tales como áreas verdes, árboles, arbustos y edificios (Figura 7). No obstante, la mayoría de localizaciones se dieron en las áreas más arboladas en los alrededores de la casa Popenoe (Figura 7).

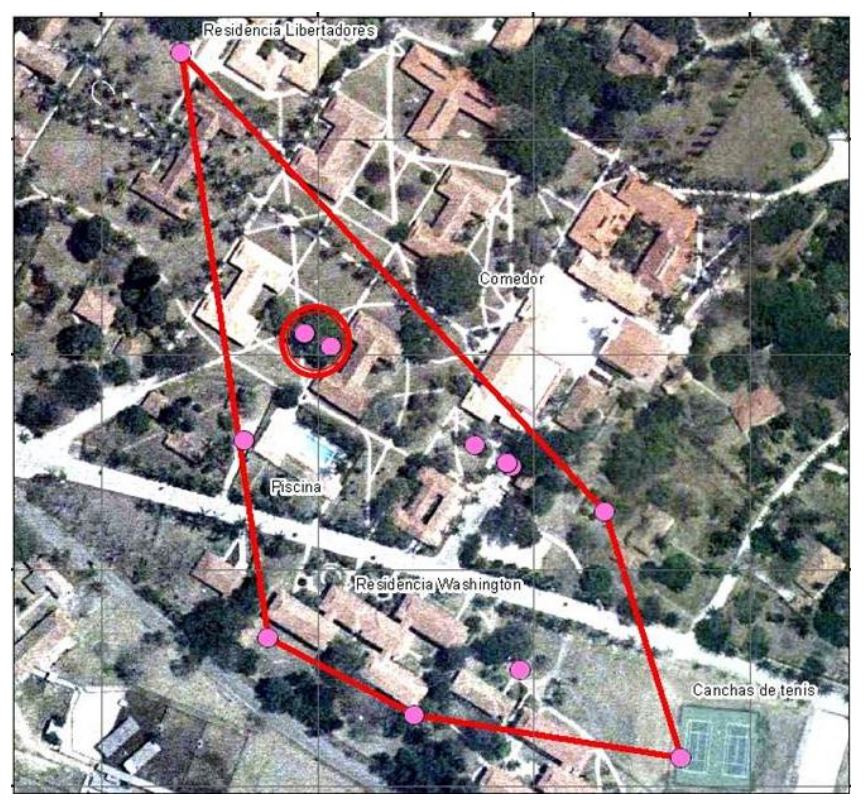

Figura 5. Polígono de frecuencias y localizaciones del zorrillo (Mephitis macroura) nombrado Leuca en el campus de la Escuela Agrícola Panamericana, Zamorano, Honduras, 2009.

Otras observaciones. Durante el tiempo de estudio se realizaron 12 observaciones de una cantidad indeterminada de otros zorrillos dentro del ámbito de hogar de los zorrillos marcados. No se registró el peso ni el sexo de estos individuos, al no ser identificados individualmente no se puede asegurar si fue el mismo u otros zorrillos.

Durante los cuatro meses de estudio se registró la muerte de tres zorrillos a causa de atropello por vehículos en la carretera panamericana en los límites del campus de Zamorano (Figura 5). En este mismo tiempo se pudo observar a los zorrillos comer restos de comida dejados por la personas, especialmente comida chatarra cerca de los dormitorios estudiantiles (Figura 6). A finales de septiembre, se observaron dos hembras con crías, además de Leuca. Una de ellas tenía cuatro cachorros y se localizó debajo de unos arbustos. La otra hembra se vio con tres cachorros en un camino de tierra. Ambas hembras se encontraron dentro del ámbito de hogar del zorrillo macho nombrado Mex (Figura 7).

A Lanik y Bale no se les pudo seguir rastreando debido a diferentes razones (Cuadro 1). Con los datos que se pudieron obtener se notó que dichas hembras vivían dentro del ámbito de hogar de los machos (Figura 1).

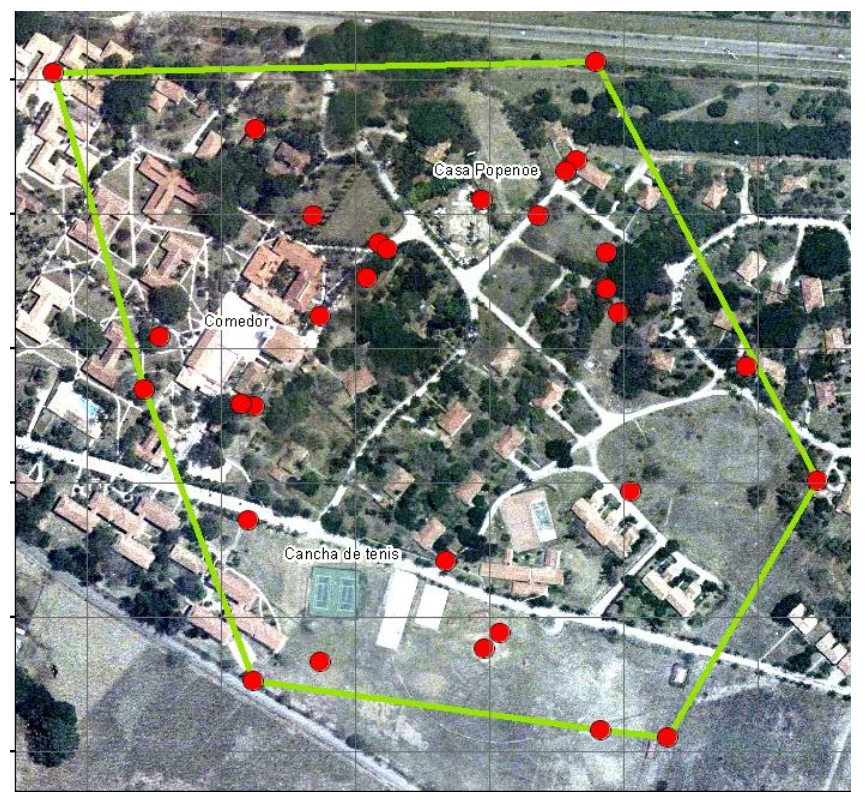

Figura 7. Polígono de frecuencias y localizaciones del zorrillo (Mephitis macroura) nombrado Mex en el campus de la Escuela Agrícola Panamericana, Zamorano, Honduras, 2009.

\section{Discusión}

El ámbito de hogar de los cuatro zorrillos fue diferente entre ellos; el más pequeño fue el de Leuca y el más grande el de Chiriquí. El ámbito de hogar de Felipe fue más pequeño que el de los demás machos, lo cual podría deberse a que es un individuo juvenil. No obstante, podrían haber otras explicaciones tales como que su territorio contenía mejores recursos o que no se le siguió el tiempo suficiente para conocer su verdadero ámbito de hogar. 
Prácticamente no existió intersección entre los polígonos de frecuencia de los ámbitos de hogar de los dos machos adultos (Figura 1). El ámbito de hogar de estos machos fue mayor que el de la hembra, al menos durante el tiempo de estudio, similar a lo determinado para Spilogale putorius en Arkansas, Estados Unidos (McCullough y Fritzell, 1984).

Al final del periodo de estudio, la hembra comenzó a moverse más lejos de su madriguera, por lo que quizá su ámbito de hogar era mayor ya que se desplazaría más en el periodo no reproductivo. Sería necesario, además, tener datos de otras hembras para determinar si en realidad los machos tienen mayores ámbitos que las hembras.

La curva del área acumulada de Chiriquí (Figura 2) se acercó a una asíntota cuando se obtuvieron más ubicaciones. Una asíntota en la curva indica que se ha llegado a un punto de estabilización del área del ámbito de hogar, ya que por más puntos que se siguieran tomando el área del ámbito de hogar no aumentaría significativamente. No obstante, el tamaño del ámbito de hogar podría variar si se incluyen factores como época de reproducción y cambio estacional. Ello implica que se amerita darles seguimiento a estos individuos para alcanzar resultados más concluyentes. Lo mismo se puede decir de Chiriquí (Figura 2) así como de los demás individuos, con excepción de Leuca (Figura 3). La curva de Leuca continuaría creciendo debido a que en gran parte del estudio esta hembra se encontró dentro de su madriguera cuidando a las crías; al final del estudio (septiembre 2009) ella comenzó a salir, por lo que el área de su ámbito de hogar se extendió.

El ámbito de hogar de las hembras se espera que varíe en dependencia de la disponibilidad de comida, mientras que el de los machos variará según la disponibilidad de hembras (Bixler y Gittleman, 2000). En este estudio se observó que dentro del ámbito de hogar de Mex, también se encontraba el de tres hembras. Adicionalmente, se vieron más zorrillos dentro del área de estudio, pero no fueron identificados, lo que significa que su densidad en el campus es alta. Al mismo tiempo, estos zorrillos se encontraron dentro del ámbito de hogar de los cuatro zorrillos rastreados por lo que podría haber mayor intersección de los ámbitos de hogar que la determinada (Figura 1).
Chiriquí fue el individuo con mayor ámbito de hogar (Cuadro 1) y fue localizado desde los potreros (de ganado de carne) hasta un túnel debajo de la carreta panamericana (Figura 4). Chiriquí fue localizado más veces en los muros de piedras, probablemente porque en ellos encuentra refugio y alimento. Su ámbito de hogar está atravesado por la carretera panamericana (Figura 4), lo que representa una amenaza para su vida. La muerte de zorrillos en esta carretera parece ser común ya que durante el período de estudio murieron tres zorrillos atropellados. Tres individuos adicionales murieron en esta carretera (2) y en la carretera a Güinope (1) en los límites del campus de Zamorano entre enero y abril de 2009.

Algunas veces se encontró a Felipe dentro del área de Mex y de Chiriquí (Figura 1). Felipe se localizó varias veces debajo de árboles y su madriguera se encontraba debajo de escombros de madera cerca de una estación de reciclaje (Figura 5). Este tipo de madriguera fue también utilizada por Mex que igualmente se encontró debajo de escombros de madera y otros materiales de construcción. Esto indica que los zorrillos pueden ser oportunistas y que este tipo de material de construcción es aprovechado cuando está disponible. Mex fue el zorrillo con más localizaciones ya que fue el primer individuo capturado y se encontró varias veces en áreas abiertas; además, se encontró varias veces cerca de edificios (Figura 7), quizá porque encuentra alimento que dejan las personas.

La madriguera de Leuca se encontraba debajo de un árbol grande cerca de dos residencias estudiantiles, donde crió a su camada (Figura 6). Leuca estuvo en dos madrigueras que se encontraban próximas una a la otra debajo de un camino de piedras. El $50 \%$ de las veces que se encontró a Leuca fue en estos dos sitios, lo que indica que salía muy poco de la madriguera y cuando lo hacía era por poco tiempo. En otro estudio se pensaba que dos hembras de Mephitis mephitis tenían camadas porque permanecieron de dos a cuatro semanas en un área reducida, probablemente cerca de una madriguera de cría. Una de dichas hembras capturadas tuvo una camada de cinco cachorros (Bixler y Gittleman, 2001).

Se determinó un ámbito de hogar de 54 a 135 ha para las hembras de Spilogale putorius en Norteamérica. No obstante, los machos tuvieron 
grandes diferencias estacionales en el ámbito de hogar ya que variaron de 76 a 175 ha durante otoño e invierno y 866 ha en primavera (Lesmeister et al., 2009). En el estudio de Bixler y Gittleman (2001), el ámbito de hogar promedio de 15 zorrillos Mephitis mephitis fue 108 ha. El tamaño del ámbito de hogar de Mephitis macroura en el campus de Zamorano es menor (Figura 1) al reportado para otras especies. Estas diferencias se pueden deber a varios aspectos como la necesidad de más localizaciones o de más datos ya que el ámbito podría variar con las estaciones del año (disponibilidad de alimento y reproducción). Otra explicación para que Mephitis macroura tenga ámbitos de hogar más pequeños que otras especies, aparte de ser una especie con individuos de menor tamaño corporal, es que estos animales encuentran en el campus de Zamorano condiciones altamente favorables que les exigen menos desplazamiento para llenar sus requerimientos ecológicos básicos.

Las madrigueras encontradas en el campus de Zamorano fueron variadas, lo que significa que este recurso no es una limitante en este sitio. Los tipos de refugios y madrigueras usados por Mephitis macroura en el campues de Zamorano son similares a los usados por Mephitis mephitis en Norteamérica (Patton, 1974). Además, la presencia de espacios cerrados debajo de edificios es importante para el zorrillo, posiblemente por el ahorro de energía para construir y mantener las madrigueras, ventajas de termorregulación y reducción de riesgos de depredación (Larivière et al., 1999). Algunas construcciones humanas en Zamorano son un refugio para el zorrillo ya que proveen espacios adecuados para su alimentación, descanso, protección y crianza, elementos que, además, les permiten ahorrar energía al no tener que recorrer grandes distancias para satisfacer estas necesidades.

Finalmente, se desconoce los impactos del uso de restos de comida de humanos en la salud y en términos ecológicos de los zorrillos. La disponibilidad e importancia de este alimento no fue estudiada, pero debe de influir el patrón espacial de cada individuo y en la existencia de varios individuos en la misma área (Bixler y Gittleman, 2001). Esta puede ser una explicación del porqué existe intersección entre los polígonos de frecuencia de los zorrillos estudiados (Figura 1) y cómo las actividades humanas pueden influir en el comportamiento de estos zorrillo.
El ámbito de hogar de estos zorrillos podría depender del sexo y de la edad de los zorrillos, puesto que se encontraron diferencias entre los ámbitos de hogar de los machos adultos, de un individuo joven y de una hembra. No obstante, esta información no es concluyente por provenir de pocos individuos por lo que se debe generar más información para alcanzar mejores conclusiones. Sería conveniente estudiar el ámbito de hogar de la especie durante las diferentes estaciones del año, ya que los estudios realizados con otras especies de zorrillo muestran diferencias en cada época. La estación seca en Zamorano es prolongada y al final de ésta la disponibilidad de agua, y posiblemente de otros recursos, puede ser baja y ser factores limitantes para los zorrillos.

Debido a que la información disponible de esta especie en la región es limitada, la información generada en este estudio es importante para el conocimiento de la ecología y conservación de la especie. Adicionalmente, se podría trabajar en la determinación del tamaño poblacional y la densidad así como otros aspectos de la ecología en el campus de Zamorano. Los individuos marcados individualmente pueden ser seguidos a través del tiempo de manera individualizada y de esa manera se podrá obtener información acerca de la longevidad de los individuos, sus hábitos de reproducción y de alimentación entre otros. Aquellos individuos que a la vez están marcados con radio collares pueden ser localizados con los fines planteados como meta en este estudio.

\section{Literatura Citada}

Bixler, A., J.L. Gittleman. 2000. Variation in home range and use of habitat in the striped skunk (Mephitis mephitis). Journal of Zoology 251(4):525-533.

Burt, W.H. 1943. Territoriality and home range concepts as applied to mammals. Journal of Mammalogy 24:346352.

Cardillo, M., Purvis, A., Sechrest, W., Gittleman, J.L., Bielby, J. y Mace, G.M. 2004. Human population density and extinction risk in the World's carnivores. PLoS Biology 2: 909-914.

Cochran, W.W. 1987. Telemetría en vida silvestre. p. 531545. In Manual de técnicas de gestión de vida silvestre. Ed. R. Rodríguez Tarrés (versión en español). The Wildlife Society. Maryland. USA. 
De la Rosa, C. y C.C. Nocke. 2000. A guide to the carnivores of Central America. University of Texas Press, Austin. USA. 244 p.

Hwang, Y.T. y S. Larivière. 2001. Mephitis macroura. Mammal species no.686:1-3.

Larivière, S. L.R. Walton y F. Messier. 1999. Selection by Striped Skunks (Mephitis mephitis) of farmsteads and buildings as denning sites. The American Midland Naturalist 142(1):96-101.

Lesmeister, D.B., M.E. Gompper y J.J. Millspaugh. 2009. Habitat selection and home range dynamics of Eastern Spotted Skunks in the Ouachita Mountains, Arkansas, USA. The Journal of Wildlife Management 73(1):1825.

McCullough, C.R. y E.K. Fritzell. 1984. Ecological observations of eastern spotted skunks on the Ozark Plateau. Transactions, Missouri Academy of Science $18(1): 25-32$

Mora, J.M. 2000. Los mamíferos silvestres de Costa Rica. San José, Costa Rica. Editorial Universidad Estatal a Distancia. 240 p.
Patton, R. F. 1974. Ecological and behavioral relationships of the skunks of Trans Pecos Texas. Tesis de doctorado. Texas A\&M University. Texas, Estados Unidos. $199 \mathrm{p}$.

Rabinowitz, A. 2003. Manual de capacitación para la investigación de campo y la conservación de la vida silvestre. Wildlife Conservation Society. Bolivia. 310 p.

Reid, F.A. 2009. A Field Guide to the Mammals of Central America \& Southeast Mexico. 2.ed. New York, US. Oxford University Press. 346 p.

White, G.C. y R.A. Garrott. 1990. Analysis of Wildlife Radio-Tracking Data. San Diego, CA, US. Academic Press. 383 p.

Recibido para publicación el 21 de noviembre de 2011.

Aceptado para publicación el 27 de noviembre de 2012. 